\title{
Decision Support System for Achieving Scholarship Selection by Using Profile Matching Method
}

Rani Irma Handayani1 ${ }^{\text {st }}$

STMIK Nusa Mandiri Jakarta

rani.rih@nusamandiri.ac.id

\author{
Triningsih2 $2^{\text {nd }}$ \\ Universitas Bina Sarana Informatika \\ triningsih.tnh@bsi.ac.id
}

Submitted: Mar 19, 2020

\author{
Melia Putri $3^{\text {rd }}$ \\ STMIK Nusa Mandiri Jakarta \\ meliaputri02@gmail.com
}

\author{
Accepted: Apr 1, 2020
}

Published: Apr 1, 2020

\begin{abstract}
Learning is one of the obligations of students to do in every school activity where they study. However, sometimes many students are less able to digest the subject matter delivered by the teacher. Therefore, the school held a scholarship program for outstanding students. In order to motivate students to study harder. Achievement scholarships are given with the aim of motivating students to study harder. Currently the scholarship is not right on target because it is still done manually and it is not clear the criteria for a student to get an achievement scholarship. To conduct an assessment in awarding scholarships to high achieving students use a decision support system to help solve a problem. For this reason, to conduct an assessment in the awarding of scholarships, a decision support system using the Profile Matching method is used. Profile Matching method is one of the methods used in decision making. In this study, there are several aspects of the assessment for awarding achievement scholarships, namely the KKM Aspect, the Attendance Aspect, the Behavior Aspect, the Craft Aspect or the Discipline, the Neatness Aspect.
\end{abstract}

Keywords - Profile Matching, Decision Support System, Achievement Scholarships

\section{INTRODUCTION}

Bina Insan Mandiri Vocational School is one of the High Vocational Schools that provides achievement scholarships with the aim of motivating students to study harder. Scholarships are aids to individuals to continue the education being pursued (Sari 2018). Not all prospective scholarship recipients will receive a scholarship, only candidates who meet the established criteria will receive the scholarship ( rani irma Handayani 2017)

At present the awarding of scholarships is still done manually and subjectively to the lengthy process of determining the scholarship (Oktavia 2018). Due to limited time and limited ability to see all aspects accurately often leads to mistakes in decision making (R. I. Handayani 2015) 
Therefore, an assessment is needed for awarding scholarships to high achieving students by using a decision support system to help solve a problem (Saryoko, Aziz, and Nurmalia 2020). Methods that can be used in assessing scholarships for high achieving students, namely the Profile Matching method. This method is used to avoid mistakes in decision making (Budi Sudrajat 2018). This profile matching method can also facilitate schools in decision-making (Indriyani et al. 2019). Decision making is based on aspects or criteria that have been determined (Apriana 2016)

\section{LITERATURE REVIEW}

\section{A. Decision support system}

Decision support system (DSS) is an interactive information system that provides information, modeling, and manipulating data (Kusrini 2007)

\section{B. Profile Matching}

In the profile matching process in outline is a process of comparing between each criteria for each assessment in a proposal research proposals submitted so the difference in scores is known (also called GAP (Gross Across Product)), the smaller the GAP produced, the greater the weight value which means it has a greater chance for eligibility / graduation priority. (Apriana 2018)

The steps in profile matching are:

1. Determine the mapping variables Competency gaps determine the aspects that will be used in processing employee grades.

2. Calculating the results of the competency Gap mapping referred to by the Gap here is the different profiles between employees and the expected standard profile or can be shown in the formula below:

Gap = Employee profile - Profile matching..(1).

After obtaining a gap in each employee, each employee's profile is weighted by benchmarking the gap value weight table.

Then each aspect is grouped into 2 groups, namely the Core Factor and Secondary Factor groups. Core Factor calculations are shown using the formula below:

$$
\mathrm{NCF}=\frac{\sum \mathrm{Nc}}{\Sigma \mathrm{Ic}} \ldots \ldots \ldots \ldots \text { (2) }
$$

$\mathrm{NCF}=$ The average value of the core factor

$\mathrm{NC}=$ The number of core factor values

$\mathrm{IC}=$ Number of core factor items

Meanwhile, the secondary factor calculation is indicated by the following formula:

$$
\mathbf{N S F}=\underline{\boldsymbol{\Sigma} \mathbf{N S}} \ldots \ldots \ldots \text { (3) }
$$

$\mathrm{NSF}=$ The average value of the secondary factor
NS $=$ Total number of secondary factor values IS $=$ Number of secondary factor item

After calculating the Core factors and Secondary factors, then calculate the total value based on the percentage of core and secondary that is estimated to affect the performance of each profile. Examples of calculations can be seen in the formula below:

$(\mathrm{x}) \% \mathrm{NCF}$ (Core Factor Average Value) + $(\mathrm{x}) \%$ NSF (Average Factor Secondary Value) $=\mathrm{N}$ (Total of aspects) (4)

Information :

$(\mathrm{x}) \%=$ The percent value inputted

Finally the Ranking calculation, the calculation can be shown by the formula below

Ranking $=(\mathrm{x}) \% \mathrm{~N} 1+(\mathrm{x}) \% \mathrm{~N} 2+(\mathrm{x}) \% \mathrm{~N} 3$

Information:

N1,N2,N3 : Total aspect value calculated

(x)\% : Percent value entered

\section{DISCUSSION}

In the selection of recipients of Scholarship Assessment for Student Achievement in West Jakarta Bina Insan Mandiri Vocational School by using the profile matching method there are several aspects assessed and from these aspects there are sub criteria, namely as follows:

Table 1. Aspects of Providing Scholarships for Outstanding Students

\begin{tabular}{|l|}
\hline \multicolumn{1}{|c|}{ Scholarship aspects } \\
\hline 1. KKM Aspects \\
\hline a. Mathematics \\
\hline b. Indonesian \\
\hline c. English \\
\hline d. Natural science \\
\hline e. Social studies \\
\hline $\begin{array}{l}\text { f. } \text { Computer Skills and Information } \\
\text { Managemen }\end{array}$ \\
\hline 2. Aspect of Presence \\
\hline a. Punctuality in coming to school \\
\hline b. Punctuality in entering class \\
\hline $\begin{array}{l}\text { c. Punctuality in participating in ceremonial } \\
\text { activities }\end{array}$ \\
\hline $\begin{array}{l}\text { d. Punctuality in participating in practicum } \\
\text { activities }\end{array}$ \\
\hline 3. Behavior Aspects \\
\hline $\begin{array}{l}\text { a. } \text { Respect, respect the teacher or classmates } \\
\text { and keep the classroom atmosphere in } \\
\text { good condition (not making noise, } \\
\text { chatting, or laughing that has nothing to }\end{array}$ \\
\hline
\end{tabular}


do with the lesson)

b. Knock on the door, ask for permission to enter or exit the class, behave politely or friendly

c. Does not violate the rules

d. Obey all commands of the teacher while in the school environment

e. Not asking before the teacher asks

\section{Crafts / Discipline Aspects}

a. Full attention during learning (asking or answering), carrying textbooks and taking notes

b. Chase and collect tasks on time

c. The student concerned always complies with the provisions of school entrance after school and school hours

\section{Neatness aspects}

a. The students concerned always dress neatly and politely

b. Based on the following with the complete uniform attributes that have been determined from the school

c. Use black shoes and white socks

Table 2. Value of Aspect Sub Criteria

\begin{tabular}{|l|l|}
\hline \multicolumn{2}{|c|}{ Sub Criteria Value } \\
\hline 1 & Very less \\
\hline 2 & Less \\
\hline 3 & Enough \\
\hline 4 & Well \\
\hline 5 & Very good \\
\hline
\end{tabular}

\section{A. Competency Gap Mapping}

In calculating the Competency gap mapping, the gap referred to here is the difference between the scholarship acceptance profile and the student profile or can be submitted in the formula below:

\section{Gap = Student Profile - Scholarship Acceptance Profile}

Calculation of competency gap mapping is based on existing aspects. The following is the calculation of the gap for each aspect:
Table 3. Mapping Competency Gap Aspects of KKM Scholarship Acceptance

\begin{tabular}{|c|c|c|c|c|c|c|c|c|}
\hline \multirow[t]{2}{*}{ No } & \multirow[t]{2}{*}{ Studert's nams } & \multicolumn{7}{|c|}{ Varidblo } \\
\hline & & KKOI & KKOL2 & $\mathrm{KKN} B$ & KXN:4 & KKOLS & KKOL6 & \\
\hline 1 & Affith Houn & 4 & 4 & 4 & 4 & 4 & 4 & \\
\hline 2 & $\begin{array}{c}\text { Aatoni } \\
\text { Ganzwan }\end{array}$ & 3 & 4 & 4 & 4 & 4 & 3 & \\
\hline 3 & ArifisNza Ionz & 3 & 4 & 4 & 4 & 4 & 4 & \\
\hline 4 & Diner Astrieni & 4 & 4 & 4 & 3 & 4 & 4 & \\
\hline 5 & $\begin{array}{c}\text { NadiaNz } \\
\text { Maidh }\end{array}$ & 4 & 4 & 4 & 4 & 4 & 4 & \\
\hline 6 & Naz Elias Fatria & 5 & 4 & 4 & 4 & 4 & 4 & \\
\hline 7 & $\begin{array}{c}\text { RidtyEgz } \\
\text { Pratarz }\end{array}$ & 3 & 4 & 4 & 4 & 4 & 3 & \\
\hline 8 & $\begin{array}{c}\text { Saprins Potri } \\
\text { Rosita }\end{array}$ & 4 & 4 & 4 & 5 & 4 & 4 & \\
\hline 9 & $\begin{array}{l}\text { Wakyo Fobby } \\
\text { Seizuza }\end{array}$ & 3 & 4 & 4 & 4 & 4 & 5 & \\
\hline & Scbolankip & 5 & 5 & 5 & 5 & 5 & 5 & G.AP \\
\hline 1 & Affith Hiwn & -1 & -1 & -1 & -1 & -1 & -1 & \\
\hline 2 & $\begin{array}{c}\text { Aatoni } \\
\text { Ganzwin }\end{array}$ & -2 & -1 & -1 & -1 & -1 & 0 & \\
\hline 3 & ArifiaNu laura & 4 & -1 & -1 & -1 & -1 & -1 & \\
\hline 4 & Diner Aatrieni & -1 & -1 & -1 & -2 & -1 & -1 & \\
\hline 5 & $\begin{array}{c}\text { NudiaNzx } \\
\text { Madth }\end{array}$ & -1 & -1 & -1 & -1 & -1 & -1 & \\
\hline 6 & Naz Elias Fatria & 0 & -1 & -1 & -1 & -1 & -1 & \\
\hline$?$ & $\begin{array}{c}\text { RifiyEzz } \\
\text { Pratarz }\end{array}$ & -2 & -1 & -1 & -1 & -1 & -2 & \\
\hline 8 & $\begin{array}{l}\text { 3aprinz Potri } \\
\text { Rosita }\end{array}$ & -1 & -1 & -1 & 0 & -1 & -1 & \\
\hline 9 & $\begin{array}{l}\text { Wilhy Foby } \\
\text { Sotzonan }\end{array}$ & 2. & -1 & -1 & -1 & -1 & 0 & \\
\hline
\end{tabular}

Information :

KKM1 : Mathematics

KKM2 : Indonesian

KKM3 : English

KKM4 : Natural science

KKM5 : Social studies

KKM6 : Computer Skills and Information Managemen

\section{B. Determination Weight of Gap Value}

After obtaining a gap in each student, after the student profile is given a weight value by benchmarking the gap value gap table..

Table 4. Weight of Gap Value

\begin{tabular}{|c|c|c|l|}
\hline No & $\begin{array}{c}\text { Differe } \\
\text { nce }\end{array}$ & $\begin{array}{c}\text { value } \\
\text { weight } \\
\text { S }\end{array}$ & \multicolumn{1}{|c|}{ Information } \\
\hline 1 & 0 & 5 & $\begin{array}{l}\text { There is no difference } \\
\text { (competency as needed) }\end{array}$ \\
\hline 2 & 1 & 4,5 & $\begin{array}{l}\text { Individual competence is } 1 \\
\text { level / level }\end{array}$ \\
\hline 3 & -1 & 4 & $\begin{array}{l}\text { Individual competencies } \\
\text { lack 2 levels / levels }\end{array}$ \\
\hline 4 & 2 & 3,5 & Individual competence is \\
\hline
\end{tabular}




\begin{tabular}{|c|c|c|l|}
\hline & & & excess of 2 levels / levels \\
\hline 5 & -2 & 3 & $\begin{array}{l}\text { Individual competencies } \\
\text { lack 2 levels / levels }\end{array}$ \\
\hline 6 & 3 & 2,5 & $\begin{array}{l}\text { Individual competencies are } \\
\text { over 3 levels / level }\end{array}$ \\
\hline 7 & -3 & 2 & $\begin{array}{l}\text { Individual competencies } \\
\text { lack 3 levels / levels }\end{array}$ \\
\hline 8 & 4 & 1,5 & $\begin{array}{l}\text { Individual competence is } \\
\text { over 4 levels / level }\end{array}$ \\
\hline 9 & -4 & 1 & $\begin{array}{l}\text { Individual competencies } \\
\text { lack 4 levels / levels }\end{array}$ \\
\hline
\end{tabular}

Table 5. Determination of the Gap Weighting Aspects of KKM Aspects for Giving Scholarships

\begin{tabular}{|c|c|c|c|c|c|c|c|}
\hline \multirow{2}{*}{ No } & \multirow[t]{2}{*}{ Stodent's Name } & \multicolumn{6}{|c|}{ Variable } \\
\hline & & KKMI & $\mathrm{KKN} / 2$ & KKMI3 & KKMI & KKM & KKM/6 \\
\hline 1 & AfitahHussa & -1 & -1 & -1 & -1 & -1 & -1 \\
\hline 2 & Artoni Gunaran & -2 & -1 & -1 & -1 & -1 & 0 \\
\hline 3 & ArifiaNus Jauza & -2 & -1 & -1 & -1 & -1 & -1 \\
\hline 4 & Dinar Astrizni & -1 & -1 & -1 & -2 & -1 & -1 \\
\hline 5 & NadiaNur.Maidah & -1 & -1 & .1 & -1 & .1 & -1 \\
\hline 6 & Nur Elisa Fitria & 0 & -1 & -1 & -1 & -1 & -1 \\
\hline 7 & Rizky Ega Pratam & -2 & -1 & -1 & -1 & -1 & -2 \\
\hline 8 & Seprina Puri Rosita & -1 & -1 & -1 & 0 & -1 & -1 \\
\hline 9 & $\begin{array}{c}\text { Wahyo Febby } \\
\text { Setiaras }\end{array}$ & 2 & -1 & .1 & -1 & .1 & 0 \\
\hline \multicolumn{8}{|c|}{ Weight Vahe } \\
\hline 1 & Afifah Husna & 4 & 4 & 4 & 4 & 4 & 4 \\
\hline 2 & Artoni Guatran & 3 & 4 & 4 & 4 & 4 & 5 \\
\hline 3 & ArifiaNu Jauza & 3 & 4 & 4 & 4 & 4 & 4 \\
\hline 4 & Dina Astriani & 4 & 4 & 4 & 3 & 4 & 4 \\
\hline 5 & NadiaNur Maidah & 4 & 4 & 4 & 4 & 4 & 4 \\
\hline 6 & Nux Elisa Fitria & 5 & 4 & 4 & 4 & 4 & 4 \\
\hline 7 & Rizly Bga Pratam & 3 & 4 & 4 & 4 & 4 & 3 \\
\hline 8 & Ssprina Puti Rosita & 4 & 4 & 4 & 5 & 4 & 4 \\
\hline 9 & $\begin{array}{l}\text { Wahy Febby } \\
\text { Setiarian }\end{array}$ & 3 & 4 & 4 & 4 & 4 & 5 \\
\hline
\end{tabular}

\section{Calculation and Classification of Core Factors and Secondary Factors}

After determining the weight of the gap value for the five aspects. These are the $\mathrm{kkm}$ aspect, the attendance aspect, the behavioral aspect, the craft or discipline aspect, and the neatness aspect in the same way. Then each aspect is divided into two factors, namely core factor and secondary factor.

Table 6. Core Factor and Secondary Factor Values of KKM

\begin{tabular}{|c|c|c|c|c|}
\hline No & $\begin{array}{c}\text { Student's } \\
\text { Name }\end{array}$ & CF & NF & NKKM \\
\hline 1 & Afifah Husna & 4 & 4 & 4 \\
\hline
\end{tabular}

\begin{tabular}{|c|c|c|c|c|}
\hline 2 & $\begin{array}{c}\text { Antoni } \\
\text { Gunawan }\end{array}$ & 3,66667 & 4,33333 & 3,933334 \\
\hline 3 & Arifia Nur Jauza & 3,66667 & 4 & 3,800002 \\
\hline 4 & Dinar Astriani & 4 & 3,66667 & 3,866668 \\
\hline 5 & $\begin{array}{c}\text { Nadia Nur } \\
\text { Maidah }\end{array}$ & 4 & 4 & 4 \\
\hline 6 & Nur Elisa Fitria & 4,33333 & 4 & 4,199998 \\
\hline 7 & $\begin{array}{c}\text { Rizky Ega } \\
\text { Pratama }\end{array}$ & 3,66667 & 4 & 3,800002 \\
\hline 8 & $\begin{array}{l}\text { Saprina Putri } \\
\text { Rosita }\end{array}$ & 4 & 4,33333 & 4,133332 \\
\hline 9 & $\begin{array}{l}\text { Wahyu Febby } \\
\text { Setiawan }\end{array}$ & 3,66667 & 4,33333 & 3,933334 \\
\hline
\end{tabular}

\section{Scholarship Score Calculation}

The score from this process is the score of the candidate who was submitted to receive the scholarship. Determination of the score refers to the results of certain calculations. The calculation can be shown by the formula below :

\section{Score $(x) \% N k k m+(x) \% N k h d+(x) \% N k e l+(x) \% N k k+(x)$ $\%$ Nkrp}

\section{Information :}

Nkkm: KKM value

Nkhd: Presence Value

Nkel: Behavior Value

Nkk: Value of Crafts / Discipline

Nkrp: Neat Value

Table 7. Assessment Weight at SMK BINA INSAN MANDIRI

\begin{tabular}{|c|l|c|l|}
\hline \multirow{2}{*}{ No } & \multirow{2}{*}{ Assessment criteria } & \multicolumn{2}{|l|}{$\begin{array}{l}\text { Scholarship SMK } \\
\text { BINA INSAN } \\
\text { MANDIRI }\end{array}$} \\
\cline { 3 - 4 } & & Criteria & Weight \\
\hline 1 & KKM & $\checkmark$ & $40 \%$ \\
\hline 2 & Presence & $\checkmark$ & $20 \%$ \\
\hline 3 & Behavior & $\checkmark$ & $10 \%$ \\
\hline 4 & Craft / discipline & $\checkmark$ & $20 \%$ \\
\hline 5 & Neatness & $\checkmark$ & $10 \%$ \\
\hline & Amount & & $100 \%$ \\
\hline
\end{tabular}

1. Afifah Husna Score

$=(40 \% * 4)+(20 \% * 4,8)+(10 \% * 4)+(20 \% * 4,4)+$ $(10 \% * 3,7)$

$=1.6+0,96+0.4+0.88+0.37$

$=4,21$

2. Antoni Gunawan Score $=(40 \% * 3,933334)+(20 \% * 4)+(10 \% * 3)+$ 
$(20 \% * 3,7)+(10 \% * 4,7)$

$=1,5733336+0,8+0,3+0,74+0,47$

$=3,883333$

3. Score Arifia Nur Jauza

$=(40 \% * 3,800002)+(20 \% * 3)+(10 \% * 4,400002)$

$+(20 \% * 4,7)+(10 \% * 4)$

$=1,5200008+0.6+0,4400002+0.94+0,4$

$=3,900001$

4. Dinar Astriani Score

$=(40 \% * 3,86668)+(20 \% * 3)+(10 \% * 3,999998)$ $+(20 \% * 3)+(10 \% * 5)$

$=1.546672+0.6+0.3999998+0.6+0.5$

$=3,6466718$

5. Nadia Nur Maidah Score

$=(40 \% * 4)+(20 \% * 2)+(10 \% * 5)+(20 \% * 4)+$ $(10 \% * 3,3)$

$=1.6+0.4+0.5+0.8+0.33$

$=3.63$

6. Nur Elisa Fitria Score

$=(40 \% * 4,199998)+(20 \% * 4)+(10 \% * 4)+$

$(20 \% * 4,7)+(10 \% * 4)$

$=1.6799992+0.8+0.4+0.94+0.4$

$=4.219999$

7. Rizky Ega Pratama Score

$=(40 \% * 3,800002)+(20 \% * 4)+(10 \% * 4)+$

$(20 \% * 3,6)+(10 \% * 4,3)$

$=1,5200008+0,8+0,4+0,72+0,43$

$=3,870001$

8. Skor Saprina Putri Rosita

$=(40 \% * 4,133332)+(20 \% * 4)+(10 \% * 4,2)+$

$(20 \% * 3)+(10 \% * 4,1)$

$=1,6533328+0,8+0,42+0.6+0.41$

$=3,883333$

9. Skor Wahyu Febby Setiawan

$=(40 \% * 3,933334)+(20 \% * 4)+(10 \% * 3)+$

$(20 \% * 4)+(10 \% * 4)$

$=1,5733336+0,8+0,3+0,8+0,4$

$=3,873334$

Thus the one entitled to receive a scholarship is Nur Elisa Fitria who received the highest final score of 4.219999 and was ranked 1 (First) out of 9 prospective recipient students at SMK Bina Insan Mandiri West Jakarta.

Table 8. Final Results and Scores

\begin{tabular}{|c|c|c|c|}
\hline No & Student's Name & Score & Ranking \\
\hline 1 & Nur Elisa Fitria & 4,219999 & 1 \\
\hline
\end{tabular}

\begin{tabular}{|c|c|c|c|}
\hline 2 & Afifah Husna & 4,2 & 2 \\
\hline 3 & Arifia Nur Jauza & 3,900001 & 3 \\
\hline 4 & Antoni Gunawan & 3,883334 & 4 \\
\hline 5 & Saprina Putri Rosita & 3,883333 & 5 \\
\hline 6 & Wahyu Febby & & \\
\hline 7 & Setiawan & 3,873334 & 6 \\
\hline 8 & Dizky Ega Pratama & 3,870001 & 7 \\
\hline 9 & Nadia Nur Maidah & 3,63 & 8 \\
\hline
\end{tabular}

\section{CONCLUSION}

After conducting research on the granting of scholarships to outstanding students at SMK BINA INSAN MANDIRI West Jakarta, a number of conclusions were obtained, namely:

1. There are 5 aspects of the scholarship grading assessment used by SMK BINA INSAN MANDIRI West Jakarta in assessing the selection of scholarship recipients for outstanding students, namely: KKM, Attendance, Behavior, Crafts or Discipline, and Neatness.

2. The scholarship selection system application can be used as a tool for decision making while still being based on a decision support system using the Profile Matching method

\section{REFERENCES}

Apriana, Veti. 2016. "PENERAPAN METODE PROFILE MATCHING UNTUK MENENTUKAN KELAYAKAN PEMBERIAN PINJAMAN PADA BANK PERKREDITAN RAKYAT." Jurnal Moneter III(2): 144-50.

- 2018. "Penerapan Metode Profile Matching Untuk Menentukan Pemberian Reward Terhadap Pelanggan Pada Bisnis Ritel." Jurnal Pilar Nusa Mandiri 14(1): 117-22. http://ejournal.nusamandiri.ac.id/ejurnal/index.ph p/pilar/article/download/824/pdf.

Budi Sudrajat. 2018. "Pemilihan Pegawai Berprestasi Dengan Menggunakan Metode Profile Matching." Jurnal sinkron 3: 202-10.

Handayani, rani irma. 2017. "SISTEM PENDUKUNG KEPUTUSAN PEMILIHAN KARYAWAN BERPRESTASI DENGAN METODE PROFILE MATCHING PADA PT. SARANA INTI PERSADA (SIP)." Jurnal Pilar Nusa Mandiri 13.

Handayani, Rani Irma. 2015. "PEMANFAATAN APLIKASI EXPERT CHOICE SEBAGAI ALAT BANTU DALAM PENGAMBILAN KEPUTUSAN (STUDI KASUS: PT. BIT 
TEKNOLOGI NUSANTARA).” Jurnal Pilar Nusa

Mandiri 11(1): 53-59.

http://pilar.nusamandiri.ac.id/index.php/pilar/articl e/view/93 (March 9, 2018).

Indriyani, Fintri et al. 2019. "Penerapan Metode Profile Matching Sebagai Pendukung." 1(2): 2-7.

Kusrini. 2007. Andi Offset Konsep Dan Aplikasi Sistem Pendukung Keputusan.

Oktavia, Petricia. 2018. "SISTEM PENDUKUNG KEPUTUSAN SELEKSI PENERIMA BEASISWA DENGAN METODE WEIGHTED PRODUCT PADA SMP NEGERI 1 PARUNG BERBASIS WEB." 3(2): 80-86.

Sari, Retno. 2018. "Jurnal Evolusi Volume 6 No 2 - 2018." Jurnal Evolusi 6(2): 57-65.

Saryoko, Andi, Abdul Aziz, and Lia Nurmalia. 2020. "SELECTION OF EXTRACURRICULAR ACTIVITIES IN SMK INSAN AQILAH 4 JAKARTA USING PROFILE MATCHING METHOD." 16(1): 39-44. 